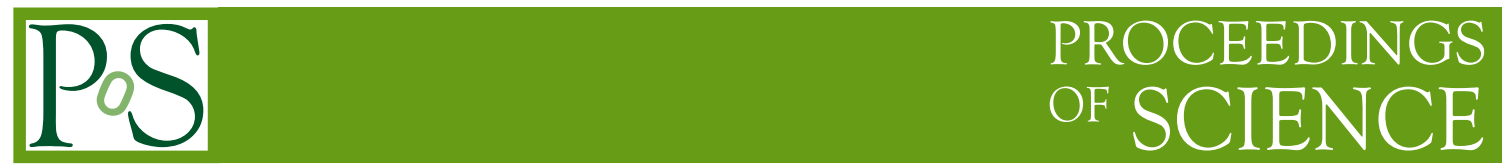

\title{
Why you should talk to preschoolers about particle physics
}

\section{Karen Gibson*}

retired, formerly of Case Western Reserve University

E-mail: karenrgibson@gmail.com

I propose that physicists consider young children in their outreach efforts to help build science literacy among the general public.

38th International Conference on High Energy Physics 3-10 August 2016

Chicago, USA

${ }^{*}$ Speaker. 
The standard model of particle physics is a remarkable theory. Entire conferences are devoted to its apparently unchecked success. Particle physicists, who are accustomed to bemoaning this very fact at these very conferences, should not forget to take appropriate pride in the wonderfully solid foundations of their field, which is why I propose that you start talking to preschoolers about the standard model.

I assure you that it is not too early. Of course, you should not begin your lesson by writing down the electroweak Lagrangian. The important thing is to introduce the words and the basic concepts. There's no reason that ideas like "quarks", "particles", "electrons", "protons", and "atoms" can't be introduced contemporaneously and that they shouldn't be introduced to the very young.

Science literacy is like any other kind of literacy. You need continual exposure to a topic to start to understand it. Think about the way we teach children to read or to perform arithmetic calculations. Parents and teachers read to children and count and add objects for them well before they are ready to fully understand these ideas. Although I am not trained in early childhood education, I am not aware of any expert in child development that would argue children should be exposed only to simple words because those are the words that they can best understand. We learn by pushing the boundaries of our understanding.

So how to do it? The importance of tactile displays cannot be overstated when working with young children. My five year old son's Montessori school teaches three and four year olds the squares and cubes of numbers. They use physical squares and cubes to demonstrate these ideas. Does he comprehend these mathematical concepts the same way I do? No, but they are part of the building blocks of his ability to do so in the future. When I have talked to preschoolers, I used cotton puffs (known as pompoms in craft stores) of different colors and sizes to talk to the children about quarks. These displays are easy and inexpensive to make and you can leave them behind in the classroom for the teachers and children to look at later.

Another crucial aspect of a successful presentation is to properly simplify your message and goals for the material. Stick to one or two basic ideas that you want to convey. Focus on those. Ask them a lot of questions to try and hold their interest. Don't talk for more than ten minutes or so, then have an activity for the class. You could have them sing a song, make up a silly dance, or draw pictures. I had each child make a new particle out of pompoms, then we glued together a particle zoo of everyone's creation. Remember, the particles don't have to make sense! When you're finished with that, come back together and recap what you talked about. Ask them questions and let them ask you some if they want.

Naturally, it is important that children's exposure to our current understanding of the physical world doesn't begin and end in pre-K. These ideas should be presented regularly throughout their school years, preferably by real, live physicists, so that by the time these same youth are in middle school or high school and are ready to comprehend it at a deeper level, it doesn't seem scary or offputting. Talk to the children of your community early and often and encourage your colleagues to do the same! It is important that they have the opportunity to meet real, live physicists if you want them to feel a personal connection to physicists later in their life. So go out and talk to children people who truly possess that very joy of discovery to which physicists so frequently lay claim and try to have some fun while you do it! Who knows, they might surprise you. Even better, you might surprise yourself! 